ISSN 0103-5150

Fisioter. Mov., Curitiba, v. 25, n. 2, p. 389-397, abr./jun. 2012 Licenciado sob uma Licença Creative Commons

\title{
Diástase dos retos abdominais em puérperas e sua relação com variáveis obstétricas
}

\author{
Diastasis of the rectus abdominis in postpartum \\ and its relation with obstetric variables
}

\author{
Ana Cristina da Nóbrega Marinho Torres Leite ${ }^{[a]}$, Kathlyn Kamoly Barbosa Cavalcanti Araújo ${ }^{[b]}$ \\ [a] Doutora em Ciências da Saúde pela Universidade Federal do Rio Grande do Norte (UFRN), Mestre em Saúde Coletiva pela \\ UEPB, docente do curso de Fisioterapia (UEPB), João Pessoa, PB - Brasil, e-mail: anacrnm@hotmail.com \\ [b] Fisioterapeuta graduada pelo Centro Universitário de João Pessoa (UNIPE), João Pessoa, PB - Brasil, e-mail: \\ kathlynkamoly@hotmail.com
}

\section{Resumo}

Introdução: Durante a gravidez, ocorrem intensas modificações no organismo materno, desencadeadas por alterações hormonais. À medida que a gestação progride, a ação hormonal em tecidos conjuntivos, associada a alterações biomecânicas para crescimento uterino podem causar o afastamento da musculatura abdominal (diástase dos músculos retos abdominais), que se estende no período pós-parto, sendo considerada diástase a separação maior que $3 \mathrm{~cm}$. Objetivos: 0 objetivo deste estudo foi confrontar a relação entre o valor das medidas da diástase dos abdominais com variáveis obstétricas em puérperas de maternidades públicas de João Pessoa. Materiais e métodos: Foram selecionadas 100 puérperas de acordo com os critérios de inclusão: idade fértil, em puerpério imediato e que durante a internação não tenham recebido atendimento fisioterapêutico para correção da diástase. Fez-se o levantamento dos antecedentes obstétricos e clínicos por meio de um questionário. Posteriormente, avaliou-se a diástase com um paquímetro digital. Para analisar a correlação significativa entre o evento e as variáveis, foi aplicado o teste do qui-quadrado. Resultados: As puérperas que apresentaram a diástase eram multíparas, multigestas, com idade entre 19 e 30 anos, tendo seus filhos por meio de partos normais, com intervalos curtos entre as gestações. Quanto à localização, houve maior incidência da diástase supraumbilical associada à separação umbilical. Conclusão: A diástase é uma condição expressiva, visto que 56 apresentaram-na. Assim, observa-se a necessidade de uma avaliação mais criteriosa das gestantes pelos profissionais de saúde para 
que haja intervenção precoce, por meio de um protocolo de atendimento, durante o pré-natal, já que traz risco para a mãe e para a futura gestação.

Palavras-chave: Diástase dos retos abdominais. Fisioterapia. Puerpério.

\section{Abstract}

Introduction: During pregnancy, enormous changes occur in the mother, triggered by hormonal alterations. As the pregnancy progresses, the hormones action in tissues to biomechanical changes associated with intrauterine growth, may cause the separation of the abdominal muscles (diastasis of the rectus abdominis muscles), which extends into the postpartum period, being considered diastasis the separation greater than $3 \mathrm{~cm}$. Objectives: The aim of this study was to compare the relationship between the value of measurements of diastasis of abdominal and obstetric variables among women in public maternity hospitals in João Pessoa. Materials and methods: 100 postpartum women were selected according to the following inclusion criteria: fertile age, being in early puerperium and not having received physical therapy to correct the diastasis during hospitalization. There was a survey of obstetric and clinic background via a questionnaire. Subsequently, we evaluated the diastasis with a caliper. To analyze the correlation between the event and the variables, we used the chi-quadrado. Results: The women who had diastasis were multiparous, aged between 19 and 30 years old, and had their children through normal deliveries, with short intervals between pregnancies. Regarding the location, there was a higher incidence of diastasis associated with supra-umbilical cord separation. Conclusion: The diastasis is an expressive condition, since 56 women had it. Thus, there is a need for a more detailed assessment of pregnant women by health professionals so that there is an early intervention, through a protocol of care during the prenatal period, since it brings risk to the mother and future pregnancy.

Keywords: Diastasis of the rectus abdominis. Physioterapy. Puerperium.

\section{Introdução}

A gravidez e o parto constituem-se em eventos fisiológicos, caracterizando-se por provocarem variadas alterações físicas e emocionais, com intuito de criar um ambiente ideal para o crescimento e desenvolvimento fetal (1-4).

As modificações durante a gestação são resultado da interação de quatro fatores: mudanças hormonalmente mediadas no colágeno e no músculo involuntário; fluxo sanguíneo aumentado para útero e rins; ampliação e deslocamento do útero em decorrência do crescimento fetal; aumento do peso corporal e mudanças adaptativas no centro de gravidade e postura $(5,6)$. 0 ganho de peso no final da gravidez tensiona a coluna lombossacra e as articulações sacroilíacas (7-9) e as transformações na postura não se corrigem espontaneamente após esse período, tornando-se uma postura adquirida após o puerpério (10).

0 útero em constante crescimento é quem sofre as transformações mais significativas na gestação, de um órgão pequeno e quase sólido a um órgão que contém o feto, a placenta e de 500 a $1.000 \mathrm{ml}$ de líquido amniótico. Embora seja um órgão muscular que não pertence ao sistema musculoesquelético em si, é a principal causa das alterações que acometem a estática e a dinâmica do esqueleto da gestante (1113), e pode, durante a gestação, causar o estiramento da musculatura abdominal, ocasionando a separação dos feixes dos músculos retos abdominais (diástase dos músculos retos abdominais - DMRA). Esta condição pode ser observada inicialmente no segundo trimestre de gestação, tendo uma incidência maior nos três últimos meses, em virtude do volume abdominal maior, assim como no pós-parto. A diástase é dita fisiológica, quando se apresenta com mais ou menos $3 \mathrm{~cm}$. Com esse grau de diástase, há retorno espontâneo às condições pré-gravídicas, sem complicações $(14,15)$.

Puerpério ou pós-parto é um período variável temporalmente, no qual as modificações locais e sistêmicas provocadas no organismo da gestante retornam ao estado pré-gravídico. Apresenta duas funções: involução e recuperação da musculatura uterina e da mucosa vaginal, com duração em média de seis a oito semanas $(16,8,4)$. Inicia-se após 
a expulsão da placenta e de membranas ovulares, prolonga-se até o momento em que todos os órgãos reprodutivos retornam ao normal, sendo dividido em imediato ( $1^{\circ}$ ao $10^{\circ}$ dia), tardio ( $11^{\circ}$ ao $45^{\circ}$ dia) e remoto (retorno às condições pré-gravídicas). As mamas são as únicas estruturas que têm seu apogeu durante o puerpério, com a liberação do colostro e formação da apojadura $(17,14,16,18)$.

A atuação da fisioterapia no pós-parto imediato visa melhorar a tonicidade dos músculos abdominais e pélvicos, informando a puérpera sobre a diástase e da importância da continuidade dos exercícios iniciados nesse período, já que o tamanho da diástase influi no tratamento fisioterapêutico $(14,6)$.

0 interesse pela temática surgiu a partir do momento em que a pesquisadora observou a escassez de referências que contemplassem esse evento, muitas vezes, subestimado pelos profissionais de saúde, deixando, assim, de ser prevenido ou minimizado.

Como a maioria dos trabalhos publicados visa à aplicação de protocolos de tratamentos para a correção da diástase, procuramos nesta pesquisa buscar os fatores que predispõem o aparecimento. Assim, o objetivo é confrontar a relação entre os valores das medidas da diástase dos retos abdominais com variáveis obstétricas em puérperas assistidas em maternidades públicas no município de João Pessoa.

\section{Materiais e métodos}

A pesquisa foi iniciada após a autorização dos responsáveis das maternidades. As participantes foram esclarecidas sobre o estudo, assinando um termo de consentimento. Depois de avaliadas, as mesmas recebiam orientações quanto ao posicionamento no leito e durante a amamentação, mudanças de postura, no intuito de favorecer o retorno da musculatura abdominal ao período pré-gravídico.

Este estudo levou em consideração as diretrizes da Resolução n. 196/96 do Conselho Nacional de Saúde/MS e suas Complementares, outorgada pelo Decreto n. 93.833, de 24 de janeiro de 1987, por se tratar de uma pesquisa envolvendo seres humanos.

0 presente trabalho correspondeu a uma pesquisa de campo, utilizando análise descritiva e de natureza quantitativa $(19,20)$.

A pesquisa foi realizada entre os meses de agosto e setembro de 2010, com 100 puérperas assistidas nas maternidades Frei Damião e Cândida Vargas, localizadas no município de João Pessoa, Paraíba, representando uma amostragem por acessibilidade, de acordo com os critérios de inclusão e de exclusão. Os critérios de inclusão foram: mulheres em idade fértil que estivessem vivenciando o puerpério imediato e que durante a internação não tivessem recebido atendimento fisioterapêutico para correção da diástase. Os critérios de exclusão corresponderam a: puérperas que se encontravam em estado de puerpério tardio ou remoto, bem como as pacientes que durante a internação tivessem recebido atendimento fisioterapêutico para correção da diástase, assim como a desistência de participação da pesquisa.

Para a coleta dos dados, utilizou-se um questionário com pontos referentes à caracterização sociodemográfica e gineco-obstétrica, exame físico (durante o qual foi realizada a análise do músculo reto abdominal) e dados antropométricos, com a finalidade de traçar um perfil do grupo. Eram avaliadas somente as puérperas que já estivessem com, pelo menos, um dia após o parto. A posição da puérpera para avaliação da diástase é em decúbito dorsal, com joelhos e quadris flexionados, pés apoiados na cama e braços ao longo do corpo. Nessa posição é solicitada a flexão anterior do tronco até que o ângulo inferior da escápula esteja fora do leito. Logo em seguida, o examinador deve palpar os limites das bordas mediais dos MRA para, depois, posicionar o paquímetro para a aferição precisa nas regiões umbilicais, supraumbilicais e infraumbilicais $(21,14,16)$. 0 paquímetro digital é um instrumento de precisão que obedece às normas internacionais para auxiliar na medição da DMRA $(22,23,24)$. Foi utilizado um da marca Western, com display LCD e indicação de medida em milímetros ou polegadas (Figura 1). Os resultados em milímetros foram transformados em centímetros: $10 \mathrm{~mm}=1 \mathrm{~cm}$. Foi utilizada, também, fita métrica para medição da altura das pacientes.

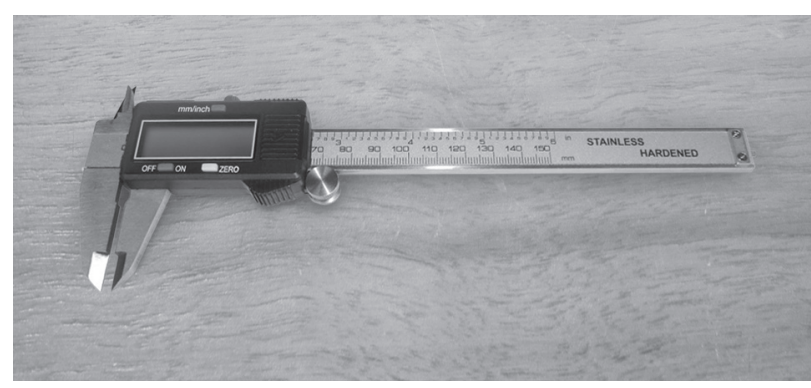

Figura 1 - Paquímetro digital Fonte: Acervo das autoras. 
Estudaram-se as seguintes variáveis:

\section{Dados sociodemográficos:}

- Idade da paciente: sendo a mesma categorizada em $\leq 18,19-30$ e > 30 anos;

- Raça/etnia: refere-se à raça que a paciente declarou pertencer, sendo a mesma dicotomizada em branca ou não branca;

- Ocupação: foi categorizada em não remunerada (atividade do lar, estudante) e remunerada;

- Escolaridade: com base no número total de anos completos de estudo formais, sendo categorizada em $\leq 8$ anos e $>8$ anos;

- Estado civil/união: estado marital com ou sem companheiro fixo.

\section{Quanto aos dados clínicos:}

- Peso: em quilogramas, e apresentado até um número decimal;

- Altura: em metros, e apresentada até dois números decimais;

- Índice de Massa Corpórea (IMC): calculado com a fórmula de Quetelet $=$ Peso $(\mathrm{kg}) /$ Altura $\left(\mathrm{m}^{2}\right)$ e classificado em três categorias: 18,5$24,9 \mathrm{~kg} / \mathrm{m}^{2}$ (normal); $25,0-29,9 \mathrm{~kg} / \mathrm{m}^{2}$ (sobrepeso) e $\geq 30,0 \mathrm{~kg} / \mathrm{m}^{2}$ (obesidade).

\section{Fatores gineco-obstétricos:}

- Número de gestações: incluindo a atual;

- Paridade: número de partos, incluindo o atual;

- Tipo de parto: cesáreo ou vaginal;

- Intervalo entre as gestações: tempo decorrido entre as gestações.

\section{Quanto à mensuração da diástase:}

- Supraumbilical: 4,5 cm acima da cicatriz umbilical;

- Umbilical: ao nível da cicatriz;

- infraumbilical: 4,5 cm abaixo da cicatriz umbilical.

Após a coleta, foi criado um banco de dados no software Microsoft Excel 2007, posteriormente utilizados para a análise estatística, por meio da estatística descritiva e inferencial. Aplicou-se nas variáveis o teste qui-quadrado $\left(\chi^{2}\right)$, que tem como finalidade encontrar o valor de dispersão para duas variáveis nominais e avaliar a associação existente entre variáveis, sendo não paramétrico, ou seja, independe de parâmetros populacionais, como média e variância.

\section{Resultados}

Os resultados mostram que a localização da DMRA mais incidente foi a supraumbilical associada à separação umbilical, correspondendo a 33 puérperas (59\%); em seguida, as puérperas que apresentaram a diástase, tanto supraumbilical, quanto umbilical e infraumbilical, respectivamente, 13 (23\%). Nesta pesquisa, nenhuma puérpera apresentou afastamento isolado ao nível infraumbilical (Tabela 1).

No que diz respeito à relação da presença da diástase dos retos abdominais com o número de gestações, o teste qui-quadrado comprovou que há significância estatística, com incidência maior entre as primigestas (Tabela 2).

Ao analisar a relação da presença da DMRA com a variável paridade, por meio da aplicação do teste do qui-quadrado, não foi observada significância estatística. Entretanto, associando os dados correspondentes à primiparidade com multiparidade, o qui-quadrado mostrou significância; por conseguinte, a chance de a mulher multípara ter diástase é 1,13 vezes maior em relação às primíparas. Ao analisar a relação entre as multíparas com as secundíparas, concluiu-se que a chance de a mulher multípara ter diástase é 1,3 vezes maior do que as secundíparas (Tabela 2).

Ao avaliar a presença de DMRA com o tipo de parto, o teste confirmou a correlação desta variável com o evento. Por meio da oddis, verificou-se uma chance 1,85 vezes maior de uma mulher submetida ao parto normal vir a ter diástases em comparação às submetidas a parto cesáreo (Tabela 2).

Embora a maioria das puérperas tenha afirmado que o intervalo entre as gestações foi acima de

Tabela 1 - Distribuição percentual do local em que prevalece a diástase do músculo reto abdominal

\begin{tabular}{lcc}
\hline \multicolumn{3}{c}{ Diástase do reto abdominal } \\
\hline & $\mathbf{n}$ & $\%$ \\
\hline Supraumbilical & 3 & 5,3 \\
Umbilical & 7 & 12,5 \\
Supraumbilical/umbilical & 33 & 59 \\
Supra/umbilical/infra & 13 & 23 \\
Infraumbilical & 0 & 0 \\
\hline Total & 56 & 100 \\
\hline
\end{tabular}

Fonte: Dados da pesquisa. 
dois anos, essa foi a amostra que mais apresentou a diástase do reto abdominal, em comparação com as puérperas que afirmaram que o tempo entre gestações foi entre 1 a 2 anos (Tabela 3).

De acordo com a análise do teste do qui-quadrado, não há significância estatística entre diástase e peso do RN. Ao se realizar esse teste individualmente para cada uma das faixas de peso, chegou-se à conclusão de que não houve significância estatística nos RNs com menos de $2.500 \mathrm{~g}$, bem como nos que tinham mais de $4.000 \mathrm{~g}$, em relação aos que tinham entre 2.500-3.990 g (Tabela 4).

\section{Discussão}

Silva et al. (25) asseguram que não há evidência científica atual que pontue o valor numérico exato

Tabela 2 - Distribuição do percentual de número de gestações, paridade e tipo de parto em relação ao grau de diástase

\begin{tabular}{|c|c|c|c|c|c|c|c|c|c|c|}
\hline \multicolumn{11}{|c|}{ Número de gestações } \\
\hline \multirow[b]{2}{*}{ DMRA } & \multicolumn{2}{|c|}{ Primigesta } & \multicolumn{3}{|c|}{ Secundigesta } & \multicolumn{2}{|c|}{ Multigesta } & \multicolumn{3}{|c|}{ Total } \\
\hline & $\mathrm{n}$ & $\%$ & & $\mathrm{n}$ & $\%$ & n & $\%$ & & $\mathrm{n}$ & $\%$ \\
\hline$\leq 3 \mathrm{~cm}$ & 21 & 47,7 & & 9 & 20,5 & 14 & 31,8 & & 44 & 44,0 \\
\hline$>3 \mathrm{~cm}$ & 27 & 48,2 & & 10 & 17,9 & 19 & 33,9 & & 56 & 56,0 \\
\hline Total & 48 & 48,0 & & 19 & 19,0 & 33 & 33,0 & & 100 & 100,0 \\
\hline \multicolumn{11}{|c|}{ Paridade } \\
\hline & \multicolumn{2}{|c|}{ Primípara } & & \multicolumn{2}{|c|}{ Secundípara } & \multicolumn{2}{|c|}{ Multípara } & \multicolumn{3}{|c|}{ Total } \\
\hline DMRA & $\mathrm{n}$ & $\%$ & & $n$ & $\%$ & $\mathrm{n}$ & $\%$ & & $\mathrm{n}$ & $\%$ \\
\hline$\leq 3 \mathrm{~cm}$ & 24 & 54,5 & & 9 & 20,5 & 11 & 25,0 & & 44 & 44,0 \\
\hline$>3 \mathrm{~cm}$ & 27 & 48,2 & & 15 & 26,8 & 14 & 25,0 & & 56 & 56,0 \\
\hline Total & 51 & 51,0 & & 24 & 24,0 & 25 & 25,0 & & 100 & 100,0 \\
\hline \multicolumn{11}{|c|}{ Tipo de parto } \\
\hline & \multicolumn{3}{|c|}{ Normal } & & \multicolumn{2}{|c|}{ Cesárea } & & \multicolumn{3}{|c|}{ Total } \\
\hline DMRA & $\mathrm{n}$ & & $\%$ & & n & $\%$ & & $\mathrm{n}$ & & $\%$ \\
\hline$\leq 3 \mathrm{~cm}$ & 32 & & 72,7 & & 12 & 27,3 & & 44 & & 44,0 \\
\hline$>3 \mathrm{~cm}$ & 33 & & 58,9 & & 23 & 41,1 & & 56 & & 56,0 \\
\hline Total & 65 & & 65,0 & & 55 & 55,0 & & 50 & & 100,0 \\
\hline
\end{tabular}

Fonte: Dados da pesquisa.

Tabela 3 - Distribuição do percentual do intervalo entre as gestações em relação ao grau de diástase

\begin{tabular}{|c|c|c|c|c|c|c|c|c|}
\hline \multicolumn{9}{|c|}{ Intervalo entre as gestações } \\
\hline \multirow[b]{2}{*}{ DMRA } & \multicolumn{2}{|c|}{ Não } & \multicolumn{2}{|c|}{ 1-2 anos } & \multicolumn{2}{|c|}{+2 anos } & \multicolumn{2}{|c|}{ Total } \\
\hline & $\mathrm{n}$ & $\%$ & $\mathrm{n}$ & $\%$ & $\mathrm{n}$ & $\%$ & $\mathrm{n}$ & $\%$ \\
\hline$\leq 3 \mathrm{~cm}$ & 21 & 47,2 & 11 & 25,0 & 12 & 27,3 & 44 & 44,0 \\
\hline$>3 \mathrm{~cm}$ & 27 & 48,2 & 5 & 8,9 & 24 & 42,0 & 56 & 56,0 \\
\hline Total & 48 & 48.0 & 16 & 16.0 & 36 & 36,0 & 100 & 100,0 \\
\hline
\end{tabular}

Fonte: Dados da pesquisa. 
Tabela 4 - Distribuição do percentual do peso do RN em relação ao grau de diástase

\begin{tabular}{|c|c|c|c|c|c|c|c|c|}
\hline \multicolumn{9}{|c|}{ Peso do RN (gramas) } \\
\hline \multirow[b]{2}{*}{ DMRA } & \multicolumn{2}{|c|}{$\leq 2.500$} & \multicolumn{2}{|c|}{$2.500-3.999$} & \multicolumn{2}{|c|}{$>4.000$} & \multicolumn{2}{|c|}{ Total } \\
\hline & $\mathrm{n}$ & $\%$ & $\mathrm{n}$ & $\%$ & $\mathrm{n}$ & $\%$ & n & $\%$ \\
\hline$\leq 3 \mathrm{~cm}$ & 7 & 15,9 & 36 & 81,8 & 1 & 2,3 & 44 & 44,0 \\
\hline$>3 \mathrm{~cm}$ & 6 & 10,7 & 47 & 83,9 & 3 & 5,4 & 56 & 56,0 \\
\hline Total & 13 & 13,0 & 83 & 83,0 & 4 & 4,0 & 100 & 100,0 \\
\hline
\end{tabular}

Fonte: Dados da pesquisa.

para considerar uma diástase em patológica. Contudo, tais autores seguem a padronização de Noble (26), que é o único item da literatura em que se estabelecem critérios e adota-se como patológica a DMRA acima de 3,0 cm. Rodrigues et al. (27) e Souza (4) também estabelecem esse critério. Rett et al. (16) garantem que uma DMRA acima de $2,5 \mathrm{~cm}$ pode ser considerada prejudicial, pois interfere na capacidade da musculatura abdominal de estabilização do tronco e em funções como postura, parto, defecação, além da contenção visceral e estabilização lombar.

De acordo com o achado desta pesquisa (Tabela 1), houve maior incidência da diástase supraumbilical associada à separação ao nível umbilical. Segundo Dore (28), Kisner e Colby (3), é mais comum a supraumbilical, pelo fato de o reto abdominal apresentar mais de um ventre muscular, separados por três inserções tendíneas acima da cicatriz umbilical. Rodrigues et al. (27) encontraram em seu estudo a presença de diástase maior que $3,0 \mathrm{~cm}$ acima da cicatriz umbilical, respectivamente, $3,5 \mathrm{~cm}$, enquanto ao nível da cicatriz umbilical e infraumbilical a separação foi de 2,0 cm. Silva et al. (25) discordam dos autores supracitados, pois em sua pesquisa, a incidência foi maior na região umbilical (52\%), enquanto que a diástase supraumbilical correspondeu a $36 \%$.

Silva et al. (25) asseveram, ainda, que a causa de a incidência infraumbilical ser menor está relacionada à morfologia da região, visto que a linha Alba é mais forte abaixo do umbigo em virtude da aponeurose de todos os quatros músculos da parede abdominal. Both et al. (18) ressaltam que o afastamento significativo do músculo reto abdominal favorece o acúmulo de gordura abdominal, diminuindo o trânsito intestinal e resultando na constipação e no surgimento das hemorroidas. Segundo Polden e Mantle (6), em separações graves, a falta de suporte abdominal dá uma menor proteção ao feto, podendo progredir para herniação das vísceras abdominais. Conforme Aragão et al. (21), durante o trabalho de parto, diástases acentuadas diminuem a qualidade da contração abdominal que, dessa forma, interfere prejudicando $o$ ato de expulsão do feto.

Os dados da Tabela 2 discordam de Souza (4), o qual analisou a influência do número de gestações na DMRA, dividindo a amostra em três grupos: primigesta, secundigesta e multigesta. Das 72 pesquisadas, 31 eram primigestas e, destas, 6 tinham a separação maior que $3,0 \mathrm{~cm}$; 3 das 22 secundigestas também apresentaram e das 19 puérperas multigestas, o evento esteve presente em 4, concluindo que as secundigestas formaram o grupo que apresentou a menor incidência de diástase (13\%). As multigestas tiveram o maior índice de afastamento (21\%). Conforme o pesquisador, os números obtidos em seu estudo sugerem a inexistência de relação entre o número de gestações bem-sucedidas com a incidência de diástase de reto abdominal e uma possível relação da individualidade tecidual como determinante do evento.

Freitas (2), Souza (4), Mesquita et al. (14), assim como Polden e Mantle (6), concordam que a multiparidade é um fator que predispõe ao afastamento do músculo reto abdominal por levar a uma maior distensão abdominal durante a gravidez. Freitas (2) acrescenta que, numa gestante multípara, a proporção é maior de abertura dos retos, pois sua musculatura já foi exigida com gestações anteriores.

Em oposição aos resultados da pesquisa apresentados na Tabela 2, Rett et al. (16) analisaram a incidência da diástase entre primíparas (227) e multíparas (240); destas, respectivamente, 154 e 163 apresentaram diástase. Foi observado, também, que as pacientes multíparas tinham uma idade 
significativamente superior $(\mathrm{p}=0,001)$ em relação às primíparas, o que, para eles, pode ser considerado um fator que corrobora com a manifestação do evento, visto que ao realizarem a revisão de literatura, a DMRA esteve presente nas que tinham idade mais avançada, maior número de gestações e partos anteriores. Eles expõem, porém, a dificuldade de se encontrar trabalhos que tenham comparado a DMRA de primíparas e multíparas no pós-parto imediato, limitando sua discussão. Sousa et al. (29) também encontraram, em sua pesquisa com 30 puérperas, a relação significativa entre a multiparidade e a diástase, apresentando uma maior relação ao aumento de DMRA, quando comparada ao grupo primigestas/ secundigestas $(\mathrm{p}=0,003)$.

Segundo Silva (24), a presença do afastamento maior que $3,0 \mathrm{~cm}$ entre os bordos do músculo reto abdominal é preocupante, pois pode ter recidivas e agravar nas gestações subsequentes.

De acordo com a Tabela 2, o afastamento do músculo reto abdominal prevaleceu em 33 pacientes com parto normal $(58,9 \%)$, em comparação às 23 $(48,1 \%)$ que apresentaram a diástase e foram submetidas à cesariana. Não há na literatura nenhum estudo que avalie a incidência da DMRA entre pacientes submetidas à cesariana e as que tiveram parto normal. 0 parto cesariano é um critério de exclusão de participação dos estudos de Both et al. (18) e Mesquita et al. (14). Não obstante, a diástase tem uma presença maior em partos normais. Both et al. (18) e Dore (28) asseveram que se esse evento não ocorreu durante a gestação, pode surgir durante a segunda fase do trabalho de parto, quando a pressão intra-abdominal aumenta (manobra de valsava), fazendo com que o útero seja empurrado para fora, contra a parede abdominal.

Silva et al. (25) investigaram, com o uso da eletromiografia de superfície, se a diástase do músculo reto abdominal interferia na fase expulsiva do trabalho de parto. A amostra foi constituída por 24 primíparas na primeira fase do trabalho de parto, na faixa etária de 19 a 29 anos, com IMC adequado para a idade gestacional. A medição da DMRA foi obtida quando a paciente ainda se encontrava no primeiro estágio do trabalho de parto. No início do período expulsivo, os eletrodos de superfície foram posicionados sobre o músculo reto abdominal, $5 \mathrm{~cm}$ superior e 3 $\mathrm{cm}$ lateralmente à cicatriz umbilical. Foi solicitado que a paciente permanecesse em repouso durante 10 segundos e os sinais eletromiográficos relativos a esse tempo foram armazenados. Posteriormente, os sinais eletromiográficos foram captados (por 15 minutos) quando a paciente começou espontaneamente os esforços expulsivos do segundo estágio do trabalho de parto. Dentre eles, o maior esforço abdominal, captado durante três segundos, foi utilizado para a análise dos dados. Como resultado, houve uma correlação negativa entre a diástase umbilical e a atividade eletromiográfica do músculo, o que pode refletir em um comprometimento na geração de força dessa musculatura durante o esforço excessivo, afetando a fase expulsiva do parto.

Os dados expostos na Tabela 3 não condizem com Both et al. (18), os quais relatam que gestações muito próximas podem constituir um fator que contribui para o aparecimento de uma separação significativa do reto abdominal, uma vez que os músculos não têm o tempo ou não realizam os exercícios necessários para serem reparados e recolocados no lugar antes que ocorra outra gravidez.

Aragão et al. (21) analisaram a relação entre a medida umbilical e o número de gestações, sendo observada uma correlação positiva e significativa. De acordo com suas pesquisas, necessita-se de pelo menos 1 (um) ano de intervalo entre as gestações para que a musculatura abdominal retorne às condições normais. Borges e Valentin (30) afirmam que os músculos abdominais podem levar seis semanas para retornar ao estado pré-gestacional e seis meses até que a força total retorne.

Avaliando-se os resultados obtidos na Tabela 4, as mães que tiveram RN com mais de $4 \mathrm{~kg}$, exatamente 3 , tiveram a diástase. De acordo com Polden e Mantle (6), Mesquita et al. (14) e Freitas (2), a macrossomia do RN (peso maior que $4.000 \mathrm{~g}$ ) é um fator de risco para o aparecimento de uma diástase acima de $3,0 \mathrm{~cm}$.

Silva (23) avaliou a relação da diástase com o peso do RN, e estes tinham um peso mínimo de 1.945 gramas e máximo de 4.000 gramas. Em seu estudo, houve correlação positiva e significante $(\mathrm{p}<0,05)$ entre o peso dos recém-nascidos $(p=0,003)$ e a separação que não regrediria espontaneamente após o parto. De acordo com a pesquisadora, a ocorrência se deve a um maior estiramento da parede anterolateral do abdômen e da linha Alba ao serem pressionados pelo útero e o seu conteúdo, além de fazer com que o período expulsivo do trabalho de parto seja mais prolongado e exigir um esforço maior da musculatura abdominal.

Segundo o estudo de Sousa et al. (27), ao avaliar o afastamento da musculatura abdominal com o peso 
do RN, chegou-se à conclusão que tem influência de $11,47 \%$ sobre a diástase $(\mathrm{p}<0,067)$.

\section{Considerações finais}

A diástase do músculo reto abdominal é uma condição que pode estar presente no último trimestre ou aparecer durante o trabalho de parto em virtude do esforço físico para expulsão do feto. De acordo com a literatura estudada, as diástases podem surgir acima da cicatriz umbilical, na cicatriz e, menos frequentemente, abaixo desse nível. Separações maiores que 3,0 cm são consideradas prejudiciais, pois podem interferir na capacidade da musculatura abdominal de estabilização e movimento do tronco, e em funções como postura, parto, defecação, além da contenção visceral.

De acordo com os dados obtidos, chegou-se à conclusão de que mulheres em idade produtiva, entre 19 e 30 anos, multigestas, multíparas, tendo seus filhos por meio de partos normais, com intervalos curtos entre as gestações, estiveram mais propensas a apresentarem DMRA.

Foi constatada, durante o processo de construção deste trabalho, a escassez de produções científicas que relacionem a condição da diástase com variáveis, sejam elas sociais ou obstétricas. Assim, torna-se clara a necessidade de pesquisas mais abrangentes para subsidiar um tratamento pré-natal ou perinatal precoce, eficaz, baseado em evidências, apontando as possíveis sequelas a longo prazo, a fim de conscientizar os profissionais da saúde para que considerem essa anormalidade com a devida importância e criem protocolos de atendimento.

Além disso, é de extrema relevância que esses profissionais incentivem as gestantes durante as consultas de pré-natal à prática de atividade física de forma segura; não somente durante a gestação, mas no puerpério, demonstrando seus benefícios nos diversos sistemas do organismo, em particular na musculatura abdominal, pois a musculatura flácida é prejudicial às mulheres, visto que favorece o acúmulo de gordura abdominal, diminui o trânsito intestinal, resultando na constipação e no surgimento das hemorroidas. Assim, percebe-se que, embora pouco estudada, a diástase dos retos abdominais constitui uma condição extremamente complexa e abrangente.

Espera-se que a referida pesquisa crie subsídios para novos estudos sobre a temática, acrescentando qualidade de vida às mulheres.

\section{Referências}

1. Centofani MD, Costa CG, Assad MAC, Moreira ECH. Perfil das participantes do "Serviço de Atendimento Interdisciplinar à Gestante". Semina: Ciênc Biológic Saúde. 2003;24:49-54.

2. Freitas RS. CRPGP, Considerações ergonômicas no período gravídico e puerperal [monografia]. Rio de Janeiro: Universidade Veiga de Almeida; 2008.

3. Kisner C, Colby LA. Exercícios terapêuticos: fundamentos e técnicas. São Paulo: Manole; 2005.

4. Souza CB. IDRAPPIAC, Incidência de diástase de reto abdominal no período de pós-parto imediato em puérperas do alojamento conjunto do HNSC [monografia]. Tubarão: Universidade do Sul de Santa Catarina; 2006.

5. Bim CR, Perego AL, Pires H. Fisioterapia aplicada à ginecologia e obstetrícia. Cesumar. 2002;4(1):57-61.

6. Polden M, Mantle J. Fisioterapia em ginecologia e obstetrícia. São Paulo: Santos; 2000.

7. Andrade AF. EDLLIRAVDG, Estudo da dor lombar e as limitações impostas durante a realização das atividades de vida diária na gestação [monografia]. João Pessoa: Centro Universitário de João Pessoa; 2008.

8. Rezende J. Obstetrícia. Rio de Janeiro: Guanabara Koogan; 2005.

9. Ruoti RG, Morris DM, Cole AJ. Reabilitação aquática para paciente obstétrica e ginecológica. São Paulo: Manole; 2000.

10. Abilío EWA. MEMAP, Manual de exercícios para a musculatura do assoalho pélvico: um estudo a partir do período gravídico-puerperal [monografia]. João Pessoa: Centro Universitário de João Pessoa; 2008.

11. Neme B. Obstetrícia básica. São Paulo: Sarvier; 2000.

12. Tfardowski APB. RTASDG, Repercussões da terapia aquática sobre as síndromes dolorosas na gestação. [monografia]. Cascavel: Universidade Estadual do Oeste do Paraná; 2004.

13. Ziegel EE, Cranley MS. Enfermagem obstétrica. Rio de Janeiro: Guanabara Koogan; 2008.

14. Mesquita LA, Machado AV, Andrade AV. Fisioterapia para redução da diástase dos músculos retos abdominais no pós-parto. Rev Bras Ginecol Obstet. 1999; 21(5):267-72. 
15. Souza ELB. Fisioterapia aplicada à obstetrícia. Rio de Janeiro: Medsi; 2002.

16. Rett MT, Bernardes NO, Santos AM, Oliveira MR, Andrade SC. Atendimento de puérperas pela fisioterapia em uma maternidade pública humanizada. Fisioterapia e Pesquisa. 2009;15(4):361-6.

17. Mendonça D, Mendonça F. Obstetrícia: protocolos de diagnóstico e tratamento. Rio de Janeiro: Revinter; 2000 .

18. Both ACCL, Reis MM Neto, Moreira RSC. ECDRAPPAFS, Estudo comparativo da diástase do reto abdominal em puérperas praticantes de atividade física e sedentárias [monografia]. Amazônia: Universidade da Amazônia; 2008.

19. Neves JL. Pesquisa qualitativa - características, usos e possibilidades. Caderno de Pesquisa em Administração. 1996;1(3):1-5.

20. Spink P. Pesquisa de campo em psicologia social: uma perspectiva pós-construcionista. Psicol \& Soc. 2003; 15(2):18-42.

21. Aragão ASL, Jesus CS, Spínola FD. Prevalência da diástase dos músculos retos abdominais em gestantes em um hospital geral público do interior da Bahia. [Revista Digital efdeportes] 2009 [acesso 17 out. 10];13(129). Disponível em: http://www.efdeportes. com/efd129/prevalencia-da-diastase-dos-musculos-retos-abdominais-em-gestantes.htm.

22. Hannaford R, Tozer, J. An investigation of the incidence, degree, and possible predisposing factors of rectus diastasis in the immediate postpartum period. J National Obstetric Gynaecologic Group Australian Physioter Assoc. 1985;4:29-34.

23. Boxer S, Jones S. Intrarater reliability of rectus abdominis diastasis measurement using dial calipers. Australian Physioterapy. 1997;43 (2):109-13.
24. Silva MMM. Análise morfométrica dos músculos retos do abdome no puerpério imediato [dissertação]. Recife: Universidade Federal de Pernambuco UFPE; 2003.

25. Silva CB, Lemos A, Oliveira BDR. A diástase do músculo reto abdominal interfere na prensa abdominal no período expulsivo do parto? [acesso 7 set. 10]. Disponível em: http//www.portalsaudebrasil.com/ artigos/artpz1.pdf.

26. Noble E. Essential exercises for the childbearing year. Houghton Mifflin Company. 1982;8:58-63.

27. Rodrigues JC Jr, Milagres II, Botaro CA. Tratamento fisioterapêutico da diástase do músculo reto abdominal em puérperas: um estudo piloto. Rev Cient FAMINAS. 2008;Supl 1:4.

28. Dore MR. COFP, cartilha de orientações fisioterapêuticas para puérperas [monografia]. Centro Universitário de João Pessoa. João Pessoa: UNIPE; 2009.

29. Sousa CAAR, Oliveira RA, Lima ACG. Diástase dos músculos retos abdominais em puérperas na fase hospitalar. Rev Bras Fisioter. 2009;10(5):333-8.

30. Borges FS, Valentin EC. Tratamento da flacidez e diástase do reto-abdominal no puerpério de parto normal com o uso de eletroestimulação muscular com corrente de média frequência: estudo de caso. Rev Bras Fisioter. 2002;1(1):2-6.
Recebido: 07/02/2011

Received: 02/07/2011

Aprovado: 01/08/2011

Approved: 08/01/2011 